\title{
Heterogeneity in the respiratory symptoms of patients with mild-to-moderate COPD
}

This article was published in the following Dove Press journal:

International Journal of COPD

\author{
Kate $M$ Johnson' \\ Abdollah Safari ${ }^{1,2}$ \\ Wan C Tan ${ }^{3}$ \\ Jean Bourbeau ${ }^{4}$ \\ J Mark FitzGerald ${ }^{2}$ \\ Mohsen Sadatsafavi ${ }^{1,2,5}$ \\ On behalf of the Canadian \\ Cohort of Obstructive \\ Lung Disease (CanCOLD) \\ study and the Canadian \\ Respiratory Research \\ Network \\ 'Collaboration for Outcomes \\ Research and Evaluation, Faculty of \\ Pharmaceutical Sciences, University \\ of British Columbia, Vancouver, \\ BC, Canada; ${ }^{2}$ Institute for Heart \\ and Lung Health, Department of \\ Medicine, The University of British \\ Columbia, Vancouver, BC, Canada; \\ ${ }^{3}$ Centre for Heart Lung Innovation \\ (the James Hogg Research Centre), \\ St Paul's Hospital, Vancouver, BC, \\ Canada; ${ }^{4}$ Respiratory Epidemiology \\ and Clinical Research Unit, McGill \\ University Health Centre, Montreal, \\ QC, Canada; ${ }^{5}$ Centre for Clinical \\ Epidemiology and Evaluation, \\ Vancouver Coastal Health Institute, \\ Vancouver, BC, Canada
}

Correspondence: Mohsen Sadatsafavi Collaboration for Outcomes Research and Evaluation, Faculty of Pharmaceutical Sciences, University of British Columbia, Vancouver Campus, 2405 Wesbrook Mall, Vancouver, BC V6T IZ3, Canada $\mathrm{Tel}+\mathrm{I} 6048273020$

Fax +l 6048755179

Emailmsafavi@mail.ubc.ca
Background: The burden of symptoms varies markedly between patients with COPD and is only weakly correlated with lung function impairment. While heterogeneity in lung function decline and exacerbations have been previously studied, the extent of heterogeneity in symptoms and the factors associated with this heterogeneity are not well understood.

Methods: A sample of the general Canadian population $\geq 40$ years with persistent airflow limitation was followed for up to 3 years. Participants reported whether they experienced chronic coughing, phlegm, wheezing, or dyspnea during visits at 18-month intervals. We used mixedeffect logistic regression models (separately for each symptom) to assess overall heterogeneity in the occurrence of symptoms between individuals, and the proportion of variation in symptom burden explained by lung function vs all other clinical characteristics of participants.

Results: Four hundred forty-nine participants (53\% male, mean age 67 years) contributed 968 visits in total, and $89 \%$ of patients reported at least one symptom during follow-up. There was substantial heterogeneity in the individual-specific probabilities for the occurrence of symptoms. This heterogeneity was highest for wheeze and dyspnea (IQR of probabilities: $0.13-0.78$ and $0.19-0.81$, respectively). $\mathrm{FEV}_{1}$ explained $28 \%$ of the variation between individuals in the occurrence of dyspnea, $8 \%$ for phlegm, $3 \%$ for cough, and $2 \%$ for wheeze. All clinical characteristics of participants (including $\mathrm{FEV}_{1}$ ) explained between $26 \%$ of heterogeneity in the occurrence of cough to $49 \%$ for dyspnea.

Conclusion: There is marked heterogeneity in the burden of respiratory symptoms between COPD patients. The ability of lung function and other commonly measured clinical characteristics to explain this heterogeneity differs between symptoms.

Keywords: population, respiratory symptoms, chronic obstructive pulmonary disease, variability, cough, phlegm, wheeze, dyspnea

\section{Introduction}

COPD is a common inflammatory lung condition that affects close to 400 million people worldwide. ${ }^{1}$ COPD is characterized by persistent airflow limitation and symptoms such as breathlessness, chronic cough, sputum production, wheezing, and chest tightness. ${ }^{2}$ Respiratory symptoms are a major burden in many patients and are associated with an increased frequency of exacerbations, ${ }^{3}$ worse disease prognosis, ${ }^{4-6}$ lower health status, ${ }^{7,8}$ reduced quality of life, ${ }^{9}$ and higher health care resource utilization. ${ }^{10}$

The three major components of the natural history of COPD are lung function status, patterns of exacerbations, and symptom burden. ${ }^{2}$ Modern guidelines such as the Global Initiative for Chronic Obstructive Lung Disease (GOLD) appreciate the importance of all three components in disease management decisions. The GOLD guidelines recommend evaluating symptoms separately from airflow limitation and history of exacerbations in providing therapeutic recommendations. ${ }^{2}$ 
It is increasingly recognized that COPD is a heterogeneous disease. Individuals can vary markedly in their rate of lung function decline ${ }^{11}$ and frequency of exacerbations ${ }^{12,13}$ over the course of their disease. For example, COPD patients in the Lung Health Study had an annual rate of change in $\mathrm{FEV}_{1}$ that ranged from rapidly declining to modestly increasing (95\% CI: -83 to $+15 \mathrm{~mL} / \mathrm{yr}) .{ }^{11}$ Similarly, the annual rate of exacerbations observed in the MACRO clinical trial varied from 0.47 to $4.22 .{ }^{13}$ Quantifying this variation at an individual level is critical to enabling precise risk factor and disease management. ${ }^{14}$

In contrast, heterogeneity in the burden of symptoms has not received the same level of attention as other disease components. This is despite the fact that the degree of symptom impairment is increasingly recognized as an important determinant of patient management strategies, and one that is only partially dependent on the severity of airflow limitation. ${ }^{4,7,15,16}$ Previous studies have reported that patient symptoms tend to vary over the day, week, or season, ${ }^{15,17-19}$ but variation between individuals in the occurrence of symptoms has been less well characterized. Understanding the extent and drivers of this heterogeneity can help to improve our understanding of the natural history of COPD and ultimately help formulate disease management strategies that provide optimal therapeutic strategies for each patient.

Using data from a population-based prospective cohort, we assessed the burden of self-reported respiratory symptoms in patients with persistent airflow limitation in order to
1) characterize variation in the occurrence of symptoms between individuals and 2) determine the proportion of between-individual variability in symptoms that can be explained by lung function vs all other observable characteristics. We hypothesized that there is high variability in the occurrence of symptoms between individuals, and that an individual's clinical and demographic characteristics explain a larger fraction of this heterogeneity than lung function alone.

\section{Methods}

We used data from the Canadian Cohort of Obstructive Lung Disease (CanCOLD), which is a multicenter prospective longitudinal cohort study conducted across Canada. ${ }^{20}$ Individuals $\geq 40$ years old were recruited using random digit dialing and multilevel sampling to ensure representativeness of the general Canadian population. Participants were followed for a maximum of 3 years with in-person visits at baseline and at 18-month intervals. From the entire cohort of CanCOLD participants $(\mathrm{N}=1,561)$, we selected any visits in which the participant had persistent airflow limitation, defined as post-bronchodilator $\mathrm{FEV}_{1} / \mathrm{FVC}<$ lower limit of normal. ${ }^{21}$ As a result, we could have included any combination of the three study visits per participant. Participants who had airflow limitation at one visit but not at any of their subsequent visits were excluded, as their airflow limitation was not considered persistent. We also excluded participants who did not meet the clinical definition of COPD because they were asymptomatic throughout follow-up and had no smoking history. ${ }^{2}$ The sample selection procedure is shown in Figure 1.

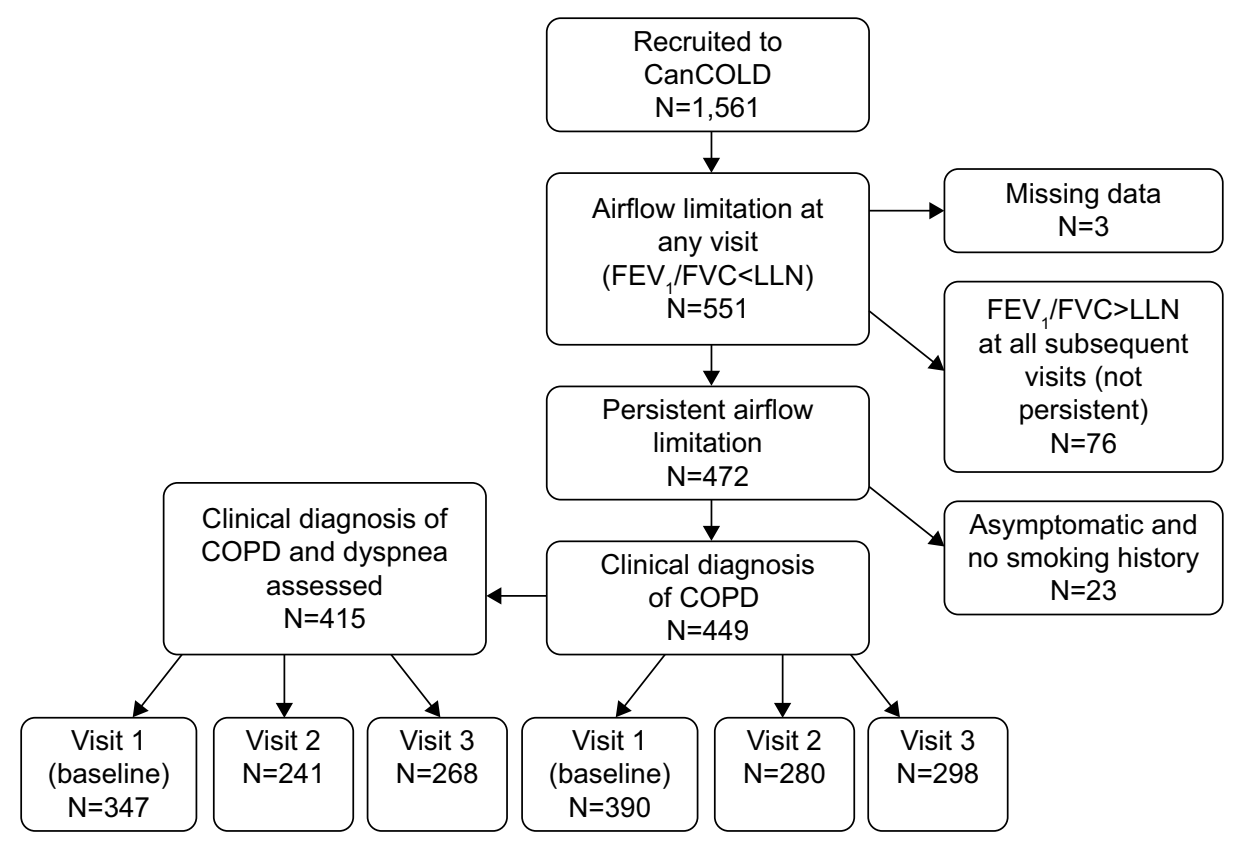

Figure I Sample selection procedure.

Abbreviations: CanCOLD, Canadian Cohort of Obstructive Lung Disease; LLN, lower limit of normal. 
Information was collected during each visit on the presence of cough, phlegm, wheeze, and dyspnea using separate questions for each symptom. Participants reported whether they 1) usually coughed in the absence of a cold; 2) brought up phlegm from the chest in the absence of a cold; and 3 ) experienced any wheezing or whistling in the chest. Dyspnea was measured using the Medical Research Council (MRC) dyspnea scale, ${ }^{22}$ which was converted to a binary variable by assuming that a score of $2-5$ indicated the presence of dyspnea. The questionnaire used to assess symptoms is reproduced in Figure S1. Dyspnea and whether the participant experienced any symptoms were assessed in a subset of the data that included 856 visits from 415 participants because 34 participants were unable to walk and therefore did not complete the MRC dyspnea test. Other variables that were assessed at each visit and included in this analysis were demographic information, smoking status and history, number of comorbidities, previous diagnosis of anxiety, major or minor depression, history of physician-diagnosed COPD (including emphysema and chronic bronchitis), and history of physician-diagnosed asthma, all self-reported using validated questionnaires with a recall period spanning the length of time between visits. ${ }^{20}$ Participants also reported the frequency and type of all respiratory-related medication use, and previous exacerbations of any severity, ${ }^{2}$ both with a 12-month recall period.

\section{Statistical analysis}

We used separate mixed-effect logistic regression models for cough, phlegm, wheeze, dyspnea, and any symptoms to model heterogeneity. A random effect term captured the variability among individuals (heterogeneity) that was not attributable to the independent variables in the model. We initially determined the total heterogeneity in the occurrence of symptoms using an intercept-only randomeffects logistic regression model for each symptom (the null model, ie, no independent variables). We used this model to determine the individual-specific probability of experiencing each symptom, and estimated the IQR $(25 \%-75 \%)$ of probabilities to measure heterogeneity in the occurrence of symptoms.

We subsequently assessed the proportion of the total heterogeneity in symptoms that could be explained by all measured characteristics of individuals. For this, we included patient age, sex, body mass index (BMI), ethnicity, number of comorbidities, diagnosis of anxiety or minor/major depression, smoking status, pack-years of smoking, any exacerbations in the past 12 months, medication possession ratio (MPR) ${ }^{23}$ for all respiratory-related medications, post-bronchodilator $\mathrm{FEV}_{1}$, previous diagnosis of asthma, and COPD diagnosis status as independent variables in each model (the full models). In order to determine the variance explained by the independent variables (ie, participants' measured characteristics), we calculated the proportion of the estimated variance in the random effect of the full model for each symptom (with all the independent variables), compared to the estimated variance in the random effect of the null model with no independent variables. ${ }^{24}$ We repeated this process using a reduced model with $\mathrm{FEV}_{1}$ as the only independent variable (as opposed to the full model) to determine the proportion of total heterogeneity explained by lung function alone.

We conducted sensitivity analyses in which percent predicted FEV 1 and GOLD grade were used in place of FEV as indicators of lung function (collinearity prevented these variables from being included in the model at the same time). Seasonality was not included in the main analysis because the recall period was $>1$ year and therefore spanned all seasons; however, season was assessed in a sensitivity analysis to account for the possibility that patients were more likely to recall their recent symptom burden (which could be affected by the current season). All analyses were performed in SAS (version 9.4, 2016).

\section{Ethics approval and informed consent}

Ethics approval for CanCOLD was obtained by the respective university and institutional ethical review boards: UBC/PHC Research Ethics Board, P05-006 (Vancouver); Biomedical-C Research Ethics Board, BMC-06-002 (Montreal); UHN REB, 06-0421-B (Toronto); Capital Health Research Ethics Board, CDHA-RS/2007-255 (Halifax); Conjoint Health Research Ethics Board, ID21258 (Calgary); DMED-1240-09 (Kingston); 2009519-01H (Ottawa); Bio-REB09-162 (Saskatoon); CER20459 (Quebec City). Written informed consent was obtained from all participants prior to study entry. CanCOLD was carried out in accordance with the principles of the Declaration of Helsinki.

\section{Results}

The characteristics of participants are shown in Table 1. There were 968 visits from 449 participants in the final sample (53\% male, mean age 67 years). Ninety-one percent of participants had mild-to-moderate disease (grade I-II), $8 \%$ had severe disease (grade III), and $1 \%$ had very severe disease (grade IV) as measured by GOLD grades. ${ }^{2}$ Seventyone percent of participants with persistent airflow limitation on spirometry had not been previously diagnosed. The 
Table I Characteristics of study participants at study visits

\begin{tabular}{|c|c|c|c|}
\hline & Visit I $(n=390)$ & Visit $2(n=280)$ & Visit $3(n=298)$ \\
\hline Age & $65.3(10.3)$ & $67.1(10.1)$ & $68.2(9.5)$ \\
\hline Male (vs female) & $54.1 \%$ & $53.2 \%$ & $52.3 \%$ \\
\hline BMI & $27.3(5.2)$ & $27.3(5.1)$ & $27.3(5.1)$ \\
\hline Caucasian (vs non-Caucasian) & $97.4 \%$ & $97.1 \%$ & $98.0 \%$ \\
\hline \multicolumn{4}{|l|}{ Comorbidities $^{\mathrm{a}}$} \\
\hline 0 comorbidities & $59.2 \%$ & $53.2 \%$ & $54.4 \%$ \\
\hline I comorbidity & $32.3 \%$ & $33.6 \%$ & $26.8 \%$ \\
\hline$\geq 2$ comorbidities & $8.5 \%$ & $13.2 \%$ & $18.8 \%$ \\
\hline Anxiety/depression (vs no) & $19.5 \%$ & $21.8 \%$ & $20.8 \%$ \\
\hline Smoking between visits (vs no) & $76.2 \%$ & $29.3 \%$ & $26.5 \%$ \\
\hline Lifetime pack-years smoked & $28.0(26.5)$ & $26.6(25.5)$ & $27.3(25.4)$ \\
\hline Any exacerbations ${ }^{\mathrm{b}}$ (vs no) & $7.9 \%$ & $11.1 \%$ & $15.4 \%$ \\
\hline $\mathrm{MPR}^{\mathrm{c}}$ & $79.9 \%(108.4)$ & $81.5 \%(112.4)$ & $79.3 \%(110.3)$ \\
\hline $\mathrm{FEV}_{1}(\mathrm{~L})$ & $2.1(0.8)$ & $2.1(0.8)$ & $2.1(0.7)$ \\
\hline$\%$ Predicted FEV & $74.4(18.1)$ & $74.9(19.1)$ & $75.7(18.8)$ \\
\hline Diagnosed COPD (vs undiagnosed) & $24.6 \%$ & $28.9 \%$ & $32.2 \%$ \\
\hline Asthma (vs no) & $20.8 \%$ & $27.1 \%$ & $29.9 \%$ \\
\hline \multicolumn{4}{|l|}{ Symptoms (present vs absent) } \\
\hline Cough & $47.7 \%$ & $47.1 \%$ & $40.6 \%$ \\
\hline Phlegm & $33.8 \%$ & $31.8 \%$ & $28.2 \%$ \\
\hline Wheeze & $51.3 \%$ & $43.6 \%$ & $42.6 \%$ \\
\hline Dyspnea $^{d}$ & $53.3 \%$ & $46.5 \%$ & $44.8 \%$ \\
\hline Any symptoms ${ }^{d}$ & $83.9 \%$ & $77.2 \%$ & $74.6 \%$ \\
\hline
\end{tabular}

Notes: Means (and SDs) are reported unless otherwise indicated. aParticipants reported whether they had ever been diagnosed with coronary artery disease, hypertension, diabetes, lung cancer, stroke, and tuberculosis at each study visit. ${ }^{b} \mathrm{COPD}$ exacerbations of any severity (mild, moderate, severe) ${ }^{2}$ over the past 12 months. ${ }^{\mathrm{c}} 12$-month medication possession ratio ${ }^{23}$ for all respiratory-related medications. ${ }^{d}$ Determined for the subset of participants in which dyspnea was measured $(\mathrm{N}=4 \mathrm{I} 5)$.

Abbreviations: BMI, body mass index; MPR, medication possession ratio.

average follow-up time was 36 months; $28 \%$ of participants underwent only one study visit, and $44 \%$ of participants were assessed at all three study visits. There were 390 , 280, and 298 participants at visit 1 (baseline), 2, and 3, respectively. The characteristics of the subset of the data used to analyze dyspnea and any symptoms were very similar (Table S1).

\section{Objective I: Heterogeneity in the occurrence of symptoms}

Most participants did not report having cough, phlegm, wheeze, or dyspnea at each study visit, but only $11 \%$ of participants were completely asymptomatic throughout the study period. The asymptomatic participants tended to have mild airflow obstruction (mean of $87 \%$ predicted $\mathrm{FEV}_{1}, 18 \% \mathrm{SD}$ ). The proportion of patients who reported a given symptom at least once during follow-up ranged from $43 \%$ for phlegm (the least common symptom) to $61 \%$ for dyspnea (the most common symptom). Symptoms were generally stable within participants: $64 \%$ of participants reported the same level of cough throughout their follow-up (the least stable symptom), and $74 \%$ for phlegm (the most stable symptom).
There was substantial variation in the individualspecific probabilities for the occurrence of symptoms that were estimated from the models (Figure 2). The median probabilities of an individual experiencing cough, wheeze, and dyspnea were $0.43,0.42$, and 0.50 , respectively. In contrast, the median probability of experiencing phlegm was 0.16 and it was $>0.99$ for any symptoms. The IQR of probabilities was $0.17-0.73$ for cough, $0.03-0.53$ for phlegm, $0.13-0.78$ for wheeze, $0.19-0.81$ for dyspnea, and 0.78 to $>0.99$ for any symptoms. Median probabilities are depicted with blue lines and IQRs are depicted with gray boxes in Figure 2.

\section{Objective 2: Influence of lung function on symptom heterogeneity}

The logistic regression models revealed relatively consistent associations between patient and disease characteristics and the presence of cough, phlegm, wheeze, dyspnea, and any symptoms. Comparisons of the strength of associations across individual symptoms are shown in Figure 3, and with any symptoms in Figure 4. Lung function, sex, pack-years of smoking, BMI, and MPR were associated with most patient-reported symptoms. Lung function was most strongly 


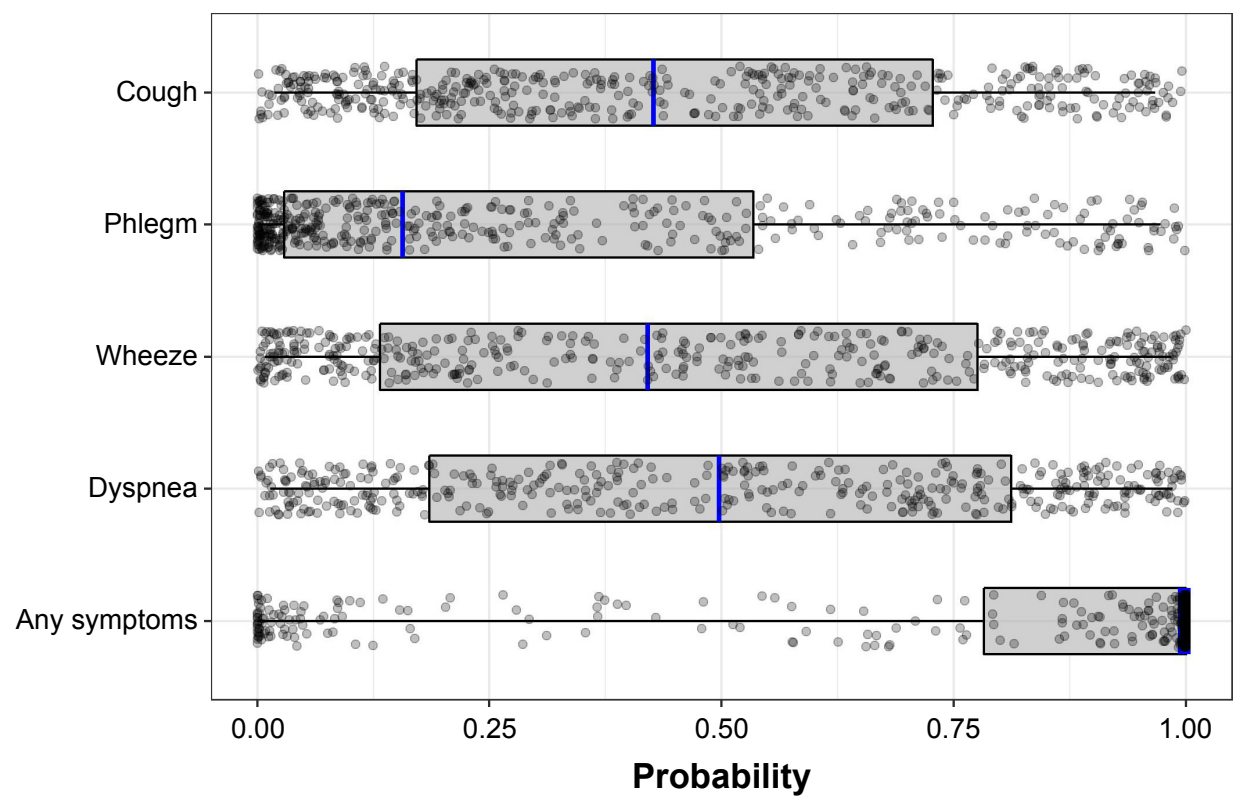

Figure 2 The distribution of individual-specific probabilities ${ }^{\mathrm{a}}$ of the occurrence of symptoms. The box spans the lower and upper quartiles $(25 \%-75 \%)$ of individuals around the median (blue line).

Notes: andividual random effects are drawn from a normal distribution with a mean of 0 and SD of the fitted random effects. The statistics shown by the boxes were determined from I,000 repetitions for each individual, and the points show the results of one repetition.

\begin{tabular}{|c|c|c|c|}
\hline Cough & OR & $95 \% \mathrm{Cl}$ & \\
\hline Visit & 0.94 & $0.73-1.2$ & $H=-1$ \\
\hline Age (10 year increase) & 0.89 & $0.66-1.19$ & $H=-1$ \\
\hline Male (vs female) & 1.62 & $0.89-2.96$ & -1 \\
\hline BMI (5 point increase) & 0.95 & $0.75-1.21$ & $H=-1$ \\
\hline Caucasian (vs non-Caucasian) & 1.18 & $0.29-4.87$ & $=$ \\
\hline 1 comorbidity (vs 0 ) & 1.12 & $0.65-1.91$ & $\vdash$ \\
\hline $2+$ comorbidities (vs 0 ) & 0.59 & $0.28-1.21$ & $10-1$ \\
\hline Anxiety/depression (vs no) & 1.53 & $0.84-2.78$ & $\rightarrow$ \\
\hline Smoking (vs nonsmoking) & 1.54 & $0.93-2.56$ & $\longmapsto$ \\
\hline Pack-years (10 PY increase) & 1.1 & $0.99-1.23$ & $\theta$ \\
\hline Any exacerbations (vs no) & 1.53 & $0.79-2.98$ & $\rightarrow$ \\
\hline MPR (20\% increase) & 1.08 & $1.03-1.14$ & H \\
\hline $\mathrm{FEV}_{1}(100 \mathrm{~mL}$ increase $)$ & 0.98 & $0.93-1.03$ & 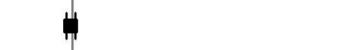 \\
\hline Diagnosed (vs undiagnosed) & 1.23 & $0.76-2$ & $\mapsto-1$ \\
\hline \multirow[t]{2}{*}{ Asthma (vs no) } & 0.75 & $0.42-1.34$ & $\mapsto--1$ \\
\hline & & & $\begin{array}{llll} & & & \\
1 & 2 & 3 & 4\end{array}$ \\
\hline
\end{tabular}

Figure 3 (Continued) 


\begin{tabular}{lll} 
B Phlegm & OR & 95\% Cl \\
\hline Visit & 0.91 & $0.68-1.23$ \\
Age (10 year increase) & 1.03 & $0.72-1.48$ \\
Male (vs female) & 4.14 & $1.89-9.03$ \\
BMl (5 point increase) & 0.97 & $0.72-1.31$ \\
Caucasian (vs non-Caucasian) & 0.85 & $0.16-4.65$ \\
1 comorbidity (vs 0) & 1.34 & $0.69-2.6$ \\
2+ comorbidities (vs 0) & 1.26 & $0.51-3.15$ \\
Anxiety/depression (vs no) & 1.46 & $0.69-3.08$ \\
Smoking (vs nonsmoking) & 2.02 & $1.1-3.74$ \\
Pack-years (10 PY increase) & 1.22 & $1.06-1.41$ \\
Any exacerbations (vs no) & 1.53 & $0.71-3.28$ \\
MPR (20\% increase) & 1.13 & $1.06-1.2$ \\
FEV (100 mL increase) & 0.93 & $0.87-0.98$ \\
Diagnosed (vs undiagnosed) & 0.82 & $0.46-1.47$ \\
Asthma (vs no) & 1.46 & $0.72-2.97$
\end{tabular}

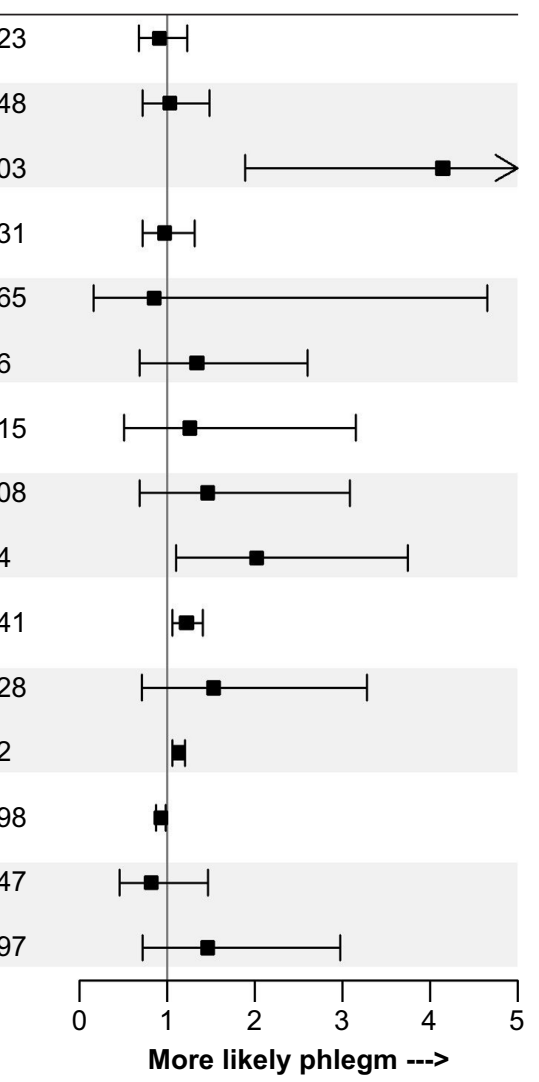

\begin{tabular}{lll} 
C Wheeze & OR & $95 \% \mathbf{~ I ~}$ \\
\hline Visit & 0.77 & $0.59-1.01$ \\
Age (10 year increase) & 0.61 & $0.43-0.86$ \\
Male (vs female) & 2.07 & $1.04-4.11$ \\
BMI (5 point increase) & 1.89 & $1.42-2.52$ \\
Caucasian (vs non-Caucasian) & 0.33 & $0.07-1.6$ \\
1 comorbidity (vs 0) & 0.91 & $0.51-1.65$ \\
2+ comorbidities (vs 0) & 0.29 & $0.12-0.67$ \\
Anxiety/depression (vs no) & 1.25 & $0.64-2.45$ \\
Smoking (vs nonsmoking) & 1.14 & $0.65-1.99$ \\
Pack-years (10 PY increase) & 1 & $0.89-1.13$ \\
Any exacerbations (vs no) & 1.87 & $0.88-3.97$ \\
MPR (20\% increase) & 1.12 & $1.06-1.19$ \\
FEV (100 mL increase) & 0.92 & $0.88-0.98$ \\
Diagnosed (vs undiagnosed) & 1.04 & $0.61-1.78$ \\
Asthma (vs no) & 3.28 & $1.69-6.36$ \\
& & \\
\hline
\end{tabular}

Figure 3 (Continued) 


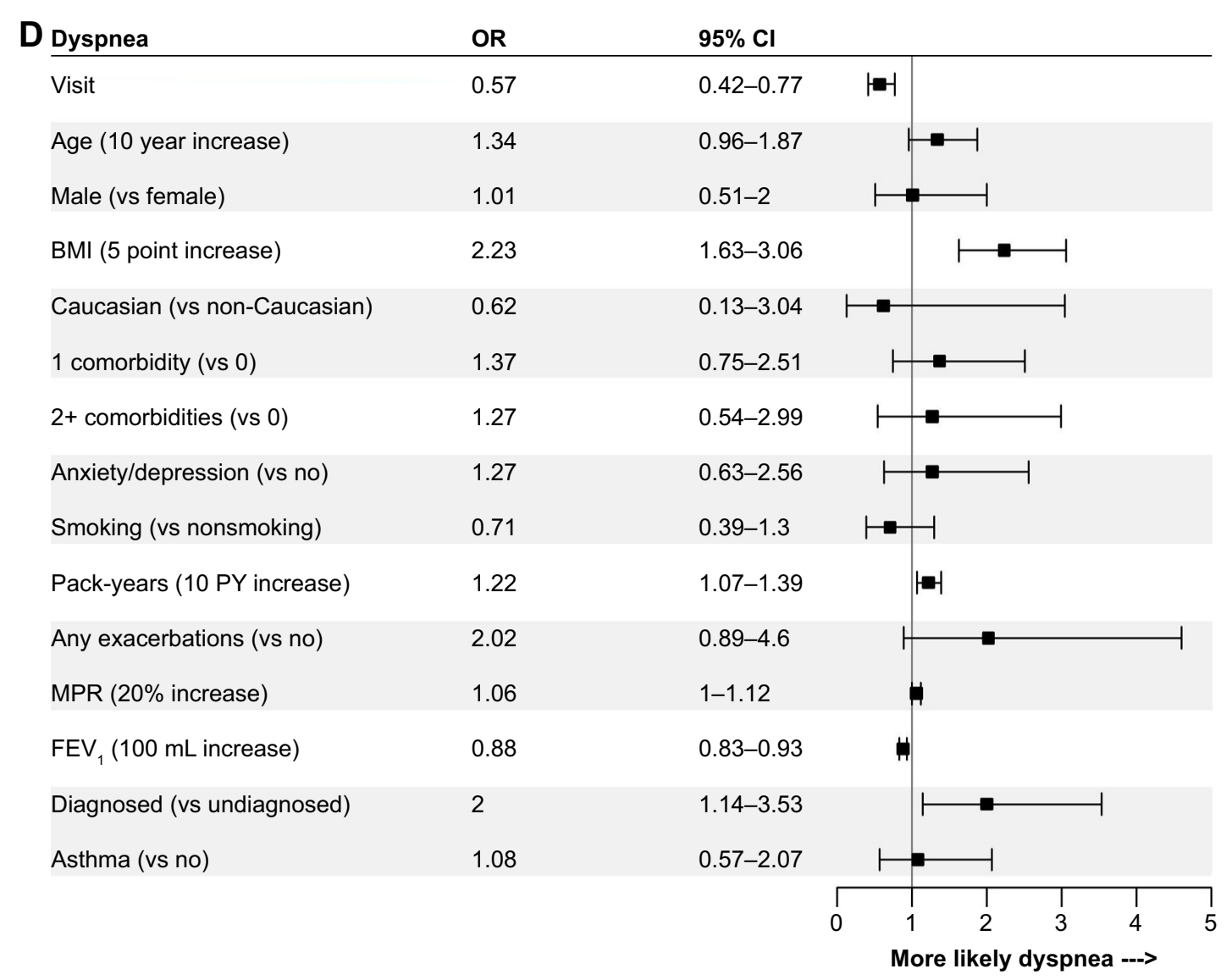

Figure 3 ORs for the associations between independent variables and the presence of (A) cough, (B) phlegm, (C) wheeze, and (D) dyspnea. Abbreviations: BMI, body mass index; MPR, medication possession ratio.

\begin{tabular}{llll} 
& OR & 95\% Cl \\
\hline Visit & 0.6 & $0.43-0.84$ \\
Age (10 year increase) & 0.66 & $0.44-1$ \\
Male (vs female) & 3.78 & $1.54-9.31$ \\
BMI (5 point increase) & 1.42 & $0.99-2.05$ \\
Caucasian (vs non-Caucasian) & 3.28 & $0.52-20.84$ \\
1 comorbidity (vs 0) & 1.54 & $0.72-3.3$ \\
2+ comorbidities (vs 0) & 0.6 & $0.22-1.63$ \\
Anxiety/depression (vs no) & 1.59 & $0.68-3.74$ \\
Smoking (vs nonsmoking) & 0.76 & $0.38-1.54$ \\
Pack-years (10 PY increase) & 1.17 & $0.99-1.38$ \\
Any exacerbations (vs no) & 1.59 & $0.48-5.32$ \\
MPR (20\% increase) & 1.13 & $1.04-1.24$ \\
FEV (100 mL increase) & 0.85 & $0.79-0.91$ \\
Diagnosed (vs undiagnosed) & 0.96 & $0.46-2$ \\
Asthma (vs no) & 1.52 & $0.66-3.49$ \\
& &
\end{tabular}

Figure 4 ORs for the associations between independent variables and the presence of any symptoms. Abbreviations: BMI, body mass index; MPR, medication possession ratio. 
Table 2 Percentage of between-individual variation in symptoms explained by individual's lung function and all measured characteristics combined

\begin{tabular}{|c|c|c|c|c|c|}
\hline & Cough (\%) & Phlegm (\%) & Wheeze (\%) & Dyspnea (\%) & Any symptoms (\%) \\
\hline $\begin{array}{l}\mathrm{FEV}_{1} \\
\text { All measured characteristics }\end{array}$ & $\begin{array}{l}3 \\
26\end{array}$ & $\begin{array}{l}8 \\
39\end{array}$ & $\begin{array}{l}2 \\
39\end{array}$ & $\begin{array}{l}28 \\
49\end{array}$ & $\begin{array}{l}78 \\
91\end{array}$ \\
\hline
\end{tabular}

Notes: ${ }^{V}$ isit, age, sex, BMI, Caucasian, comorbidities, anxiety/depression, smoking status, pack-years of smoking, any exacerbations in the past 12 months, MPR, diagnosed COPD, asthma diagnosis, and $\mathrm{FEV}_{1}$.

Abbreviations: BMI, body mass index; MPR, medication possession ratio.

associated with the presence of any symptoms (OR per $100 \mathrm{~mL}$ increase in $\mathrm{FEV}_{1}: 0.85,95 \% \mathrm{CI}: 0.79-0.91$ ), and least strongly associated with the presence of cough (OR: 0.98, 95\% CI: 0.93-1.03). These results were similar when lung function was assessed as percent predicted $\mathrm{FEV}_{1}$ or GOLD grade in sensitivity analyses (results not shown). Higher pack-years of smoking, BMI, and MPR were all associated with an increased OR of reporting most symptoms. Males were more likely than females to report the presence of phlegm (OR: 4.14, 95\% CI: 1.89-9.03), wheeze (OR: 2.07, 95\% CI: 1.04-4.11), and any symptoms (OR: 3.78, 95\% CI: 1.54-9.31). Summer (vs winter) was associated with increased reporting of cough (OR: 2.04, 95\% CI: 1.13-3.68), phlegm (OR: 2.04, 95\% CI: 1.01-4.13), wheeze (OR: 2.95, $95 \%$ CI: $1.54-5.66$ ), and any symptoms (OR: $3.13,95 \%$ CI: $1.40-6.97)$ when it was included in the sensitivity analyses.

The proportion of between-individual variation in the occurrence of symptoms that could be attributed to participants' measured characteristics (all independent variables in the full models) was $26 \%, 39 \%, 39 \%, 49 \%$, and $91 \%$, for cough, phlegm, wheeze, dyspnea, and any symptoms, respectively (Table 2 ). The proportion of variation explained by $\mathrm{FEV}_{1}$ alone ranged from $2 \%$ (for wheeze) to $78 \%$ (for any symptoms, Table 2).

\section{Discussion}

We have characterized heterogeneity in the occurrence of respiratory symptoms among patients with persistent airflow limitation and assessed the extent to which commonly measured patient and disease characteristics explained the observed heterogeneity in symptoms. Respiratory symptoms were very common in this sample despite over $90 \%$ of patients having mild-to-moderate COPD, and only $29 \%$ of them having been diagnosed with COPD. Dyspnea was the most common symptom, followed by cough and wheeze. Individual-specific probabilities for the occurrence of symptoms were highly variable between individuals and for different symptoms. The IQR of probabilities was the largest for wheeze and dyspnea, indicating greater variability between individuals in the presence of these symptoms than for cough and phlegm. For phlegm, the majority of individuals had a probability of experiencing phlegm near 0 (visible in Figure 2 as a higher density of points at the left edge of the plot). In contrast, the individual-specific probabilities for cough, wheeze, and dyspnea were more evenly spread across the range of possible values. This indicates that phlegm is more stable in nature, and that individuals who do not currently have phlegm are unlikely to report it in the future. Indeed, a pan-European study reported that daily and weekly variability in dyspnea, wheeze, and cough were higher than that for phlegm. ${ }^{25}$ Our findings extend these observations on symptom variability within individuals to variability between individuals in the occurrence of symptoms. As a result, tools for assessing COPD severity that involve the measurement of symptoms (such as the GOLD $\mathrm{ABCD}$ assessment tool) ${ }^{2}$ are likely to be more or less variable over time, depending on the symptom measured.

The proportion of heterogeneity explained by the measured characteristics of participants differed substantially between symptoms. Most heterogeneity in the occurrence of any symptoms and half the heterogeneity in dyspnea were explained by the demographic and clinical characteristics of participants included in the models. In contrast, these characteristics explained less than half the heterogeneity in phlegm and wheeze, and only a quarter of the heterogeneity in cough, indicating that other characteristics not included in our models are more important drivers of these symptoms. Cough in particular may be less predictable than dyspnea or the presence of any symptoms using many easily measured patient characteristics. Indeed, age, sex, BMI, smoking history, and lung function were weakly correlated with cough frequency ${ }^{26}$ in previous studies. Instead, cough frequency was driven by current smoking intensity and percentage of sputum neutrophils. ${ }^{26} \mathrm{~A}$ unique aspect of this study is that our assessment of heterogeneity was not limited to the patient characteristics included in our models. We quantified total between-individual variation in the burden of symptoms independent of the measured characteristics of participants. The proportion of heterogeneity that was not explained by measured characteristics highlights the potential for other 
factors, such as biologic disease markers, to improve predictions of symptom burden.

Although lung function has traditionally been regarded as the primary driver of respiratory symptoms, ${ }^{27}$ we found that $\mathrm{FEV}_{1}$ explained the majority of between-individual variation in only the occurrence of any symptoms, and a substantial minority of variation in dyspnea. This finding is in line with the observation of high symptom variability within levels of disease severity, ${ }^{28}$ and high short-term variability in symptoms that is not due to changes in lung function. ${ }^{4,16}$ Our results extend these previous studies by examining the role of $\mathrm{FEV}_{1}$ in each symptom individually. These findings suggest that lung function is the primary driver of the occurrence of any symptoms, and an important but not dominant driver of the occurrence of dyspnea, but it explains very little between-individual variation in the occurrence of phlegm, cough, and wheeze. These symptoms are expected to be more variable within levels of disease severity defined by $\mathrm{FEV}_{1}$. Our results highlight the importance of moving beyond $\mathrm{FEV}_{1}$ to incorporate other disease attributes, such as the presence of specific symptoms and exacerbation risk, when classifying disease severity. Given the large differences in the drivers of each symptom that we observed, it is likely that variation between patients in the burden of specific symptoms arises from different disease mechanisms. This can provide insights for refined COPD phenotyping.

In addition to analyzing heterogeneity in symptoms, we documented associations between symptoms and many demographic and clinical characteristics of participants. In particular, we observed substantial sex-based differences in the reporting of all symptoms apart from cough and dyspnea. Controlled for disease severity, smoking history, and other variables, male patients were over three times more likely to be symptomatic, and four times more likely to report experiencing phlegm. Whether this is a biological phenomenon, or due to gender-related differences in the experience of symptoms, ${ }^{29}$ remains to be further assessed. We also observed positive associations between the occurrence of all symptoms and MPR. The direction of this association is likely due to the long recall period over which symptoms were assessed, and reflects the underlying disease activity rather than shortterm variation in symptoms due to treatment. As a result, high treatment intensity was indicative of greater disease activity and therefore more symptomatic disease.

\section{Strengths and limitations}

Unique features of this study are its reliance on a large, nationally representative sample of the general Canadian population, the use of standardized spirometry in lung function assessment, validated questionnaires, and a long follow-up time. Our sample consisted primarily of patients with mild-tomoderate COPD, a population that is often underrepresented in large cohort studies. Further, the majority of participants in our study had undiagnosed airflow obstruction. Because patients with a higher symptom burden are more likely to seek care that leads to a diagnosis, ${ }^{30}$ samples of diagnosed patients do not accurately represent symptoms in the entire population with permanent airflow obstruction. Finally, the associations determined from conventional regressions describe the relation between patient characteristics and the presence of symptoms for an average participant. Our use of a random effect term in our models enabled us to extend these results by describing the extent to which these populationlevel associations apply to a given individual. We found that variation between individuals in the presence of any symptoms and dyspnea were reasonably well described by these population-level associations, but this was not the case for cough, phlegm, and wheeze. The assessment of variation at an individual-level is critical to fully characterize heterogeneity in the natural history of COPD, and ultimately to enable effective use of symptoms in risk prediction tools and case finding algorithms for COPD.

This study also has several limitations. Patients reported their respiratory symptoms with a recall period that spanned the length of time between study visits, which could reach a maximum of 3 years. The long duration of the recall period is likely to have resulted in inaccuracies in symptom reporting. However, our inclusion of comorbid anxiety and depression reduces the likelihood that psychological factors resulted in reporting bias. In addition, we only assessed the presence of symptoms, not their intensity. A more granular measurement of patient symptoms could provide a more nuanced assessment of symptom variability. Future studies should conduct similar analyses of symptom heterogeneity in patients with severe to very severe COPD, and in subgroups of patients defined by phenotypes or the GOLD ABCD grading system. The impact of individual symptoms on disease outcomes, such as the rate of exacerbations and the slope of lung function decline, should also be assessed. Given the tremendous heterogeneity in the burden of symptoms and their drivers, a detailed classification of patients according to their symptoms might enable better risk stratification to inform treatment decisions.

\section{Conclusion}

We assessed a sample of the general population with mostly mild-to-moderate COPD and found substantial variation in the occurrence of respiratory symptoms between individuals. 
Lung function explained the majority of between-individual variation in only the occurrence of any symptoms, and a much smaller proportion of variation in cough, phlegm, and wheeze. Commonly measured patient and disease characteristics explained very little heterogeneity in the occurrence of cough in particular. Overall, the observed differences in symptom variation may reflect the divergent etiology of symptoms associated with COPD. Defining phenotypes based on symptoms and evaluating their relation to disease outcomes are key areas of future research.

\section{Data sharing statement}

The data analyzed in the current study are not publicly available but may be made available from the CanCOLD Research Group upon reasonable request.

\section{Acknowledgments}

The authors thank participants who partook in the study and individuals in the CanCOLD Collaborative Research Group. Members of the CanCOLD Collaborative Research Group are as follows. Executive Committee: Jean Bourbeau (McGill University, Montreal, QC, Canada); Wan C Tan, J Mark FitzGerald, Don Sin (UBC, Vancouver, BC, Canada); Darcy Marciniuk (University of Saskatoon, SK, Saskatoon, Canada); Dennis E O’Donnell (Queen's University, Kingston, ON, Canada); Paul Hernandez (Dalhousie University, Halifax, NS, Canada); Kenneth R Chapman (University of Toronto, Toronto, ON, Canada); Robert Cowie (University of Calgary, Calgary, AB, Canada); Shawn Aaron (University of Ottawa, Ottawa, ON, Canada); F Maltais (University of Laval, Quebec City, QC, Canada). International Advisory Board: Jonathon Samet (Keck School of Medicine of USC, Los Angeles, CA, USA); Milo Puhan (John Hopkins School of Public Health, Baltimore, MD, USA); Qutayba Hamid (McGill University, Montreal, QC, Canada); James C Hogg (UBC James Hogg Research Center, Vancouver, BC, Canada). Operations Center: Jean Bourbeau (PI), Carole Jabet, Palmina Mancino, (McGill University, Montreal, QC, Canada); Wan C Tan (co-PI), Don Sin, Sheena Tam, Jeremy Road, Joe Comeau, Adrian Png, Harvey Coxson, Jonathon Leipsic, Cameron Hague (University of British Columbia James Hogg Research Center, Vancouver, BC, Canada). Economic Core: Mohsen Sadatsafavi (University of British Columbia, Vancouver, BC, Canada). Public Health Core: Teresa To, Andrea Gershon (University of Toronto, Toronto, ON, Canada). Data Management and Quality Control: Wan C Tan, Harvey Coxson (UBC, Vancouver, BC, Canada); Jean Bourbeau, Pei Zhi Li, Zhi Song, Yvan Fortier, Andrea
Benedetti, Dennis Jensen (McGill University, Montreal, QC, Canada). Field Centers: Wan C Tan (Vancouver PI), Christine Lo, Sarah Cheng, Elena Un, Cindy Fung, Nancy Haynes, Junior Chuang, Licong Li, Selva Bayat, Amanda Wong, Zoe Alavi, Catherine Peng, Bin Zhao, Nathalie Scott-Hsiung, Tasha Nadirshaw (UBC James Hogg Research Center, Vancouver, BC, Canada); Jean Bourbeau (Montreal PI), Palmina Mancino, David Latreille, Jacinthe Baril, Laura Labonté (McGill University, Montreal, QC, Canada); Kenneth Chapman (Toronto PI), Patricia McClean, Nadeen Audisho (University of Toronto, Toronto, ON, Canada); R Cowie and B Walter (Calgary PI), Ann Cowie, Curtis Dumonceaux, Lisette Machado (University of Calgary, Calgary, AB, Canada); Paul Hernandez (Halifax PI), Scott Fulton, Kristen Osterling (Dalhousie University, Halifax, NS, Canada); Shawn Aaron (Ottawa PI), Kathy Vandemheen, Gay Pratt, Amanda Bergeron (University of Ottawa, Ottawa, ON, Canada); Denis O'Donnell (Kingston PI), Matthew McNeil, Kate Whelan (Queen's University, Kingston, ON, Canada); François Maltais (Quebec PI), Cynthia Brouillard (Université Laval, Quebec City, QC, Canada); Darcy Marciniuk (Saskatoon PI), Ron Clemens, Janet Baran (University of Saskatoon, Saskatoon, SK, Canada). The current study was funded by a Canadian Lung Association Breathing as One Studentship Award and the Canadian Institutes of Health Research (application number 142238). The Canadian Cohort Obstructive Lung Disease (CanCOLD) study is currently funded by the Canadian Respiratory Research Network (CRRN); Industry partners: AstraZeneca Canada Ltd, Boehringer Ingelheim Canada Ltd, GlaxoSmithKline Canada Ltd, and Novartis. Researchers at RI-MUHC Montreal and Icapture Center Vancouver lead the project. Previous funding partners are the CIHR (CIHR/Rx\&D Collaborative Research Program Operating Grants 93326); the Respiratory Health Network of the Fonds de la recherche en santé du Québec (FRSQ); Industry partners: Almirall, Merck Nycomed, Pfizer Canada Ltd, and Theratechnologies. The funders had no role in study design, data collection and analysis, or preparation of the manuscript.

\section{Author contributions}

WCT and JB are co-principal investigators of the CanCOLD study. MS and KMJ formulated the current study idea. KMJ performed all data analyses and wrote the first draft of the manuscript. AS provided guidance on the statistical analysis. All authors contributed to drafting and revising the article, gave final approval of the version to be published, and agree to be accountable for all aspects of the work. 


\section{Disclosure}

The authors report no conflicts of interest in this work.

\section{References}

1. Adeloye D, Chua S, Lee C, et al. Global and regional estimates of COPD prevalence: systematic review and meta-analysis. J Glob Health. 2015;5(2):020415.

2. Global Initiative for Chronic Obstructive Lung Disease (GOLD). From the global strategy for the diagnosis, management and prevention of COPD; 2017. Available from: http://goldcopd.org. Accessed May 1, 2017.

3. Roche N, Small M, Broomfield S, Higgins V, Pollard R. Real world COPD: association of morning symptoms with clinical and patient reported outcomes. COPD. 2013;10(6):679-686.

4. Miravitlles M, Ribera A. Understanding the impact of symptoms on the burden of COPD. Respir Res. 2017;18(1):67.

5. Bridevaux PO, Gerbase MW, Probst-Hensch NM, Schindler C, Gaspoz JM, Rochat T. Long-term decline in lung function, utilisation of care and quality of life in modified GOLD stage 1 COPD. Thorax. 2008;63(9):768-774.

6. Rennard SI, Drummond MB. Early chronic obstructive pulmonary disease: definition, assessment, and prevention. Lancet. 2015;385(9979): 1778-1788.

7. Miravitlles M, Worth $\mathrm{H}$, Soler Cataluña JJ, et al. Observational study to characterise 24-hour COPD symptoms and their relationship with patient-reported outcomes: results from the ASSESS study. Respir Res. 2014;15:122.

8. Tsiligianni I, Kocks J, Tzanakis N, Siafakas N, van der Molen T. Factors that influence disease-specific quality of life or health status in patients with COPD: a review and meta-analysis of Pearson correlations. Prim Care Respir J. 2011;20(3):257-268

9. Monteagudo M, Rodríguez-Blanco T, Llagostera M, et al. Factors associated with changes in quality of life of COPD patients: a prospective study in primary care. Respir Med. 2013;107(10):1589-1597.

10. Price D, Small M, Milligan G, Higgins V, Gil EG, Estruch J. Impact of night-time symptoms in COPD: a real-world study in five European countries. Int J Chron Obstruct Pulmon Dis. 2013;8:595-603.

11. Zafari Z, Sin DD, Postma DS, et al. Individualized prediction of lungfunction decline in chronic obstructive pulmonary disease. CMAJ. 2016;188(14):1004-1011.

12. Hurst JR, Vestbo J, Anzueto A, et al. Susceptibility to exacerbation in chronic obstructive pulmonary disease. N Engl J Med. 2010;363(12): 1128-1138.

13. Sadatsafavi M, Sin DD, Zafari Z, et al. The association between rate and severity of exacerbations in chronic obstructive pulmonary disease: an application of a joint frailty-logistic model. Am J Epidemiol. 2016; 184(9):681-689.
14. Jameson JL, Longo DL. Precision medicine - personalized, problematic, and promising. N Engl J Med. 2015;372(23):2229-2234.

15. Lopez-Campos JL, Calero C, Quintana-Gallego E. Symptom variability in COPD: a narrative review. Int J Chron Obstruct Pulmon Dis. 2013; 8:231-238.

16. Miravitlles M, Menezes A, López Varela MV, et al. Prevalence and impact of respiratory symptoms in a population of patients with COPD in Latin America: the LASSYC observational study. Respir Med. 2018;134:62-69.

17. Partridge MR, Karlsson N, Small IR. Patient insight into the impact of chronic obstructive pulmonary disease in the morning: an internet survey. Curr Med Res Opin. 2009;25(8):2043-2048.

18. Espinosa de Los Monteros MJ, Peña C, Soto Hurtado EJ, Jareño J, Miravitlles M. Variability of respiratory symptoms in severe COPD. Arch Bronconeumol. 2012;48(1):3-7.

19. Donaldson GC, Goldring JJ, Wedzicha JA. Influence of season on exacerbation characteristics in patients with COPD. Chest. 2012;141(1):94-100.

20. Bourbeau J, Tan WC, Benedetti A, et al. Canadian Cohort Obstructive Lung Disease (CanCOLD): fulfilling the need for longitudinal observational studies in COPD. COPD. 2014;11(2):125-132.

21. Hankinson JL, Odencrantz JR, Fedan KB. Spirometric reference values from a sample of the general U.S. population. Am J Respir Crit Care Med. 1999;159(1):179-187.

22. Fletcher CM, Elmes PC, Fairbairn AS, Wood CH. The significance of respiratory symptoms and the diagnosis of chronic bronchitis in a working population. Br Med J. 1959;2(5147):257-266.

23. Andrade SE, Kahler KH, Frech F, Chan KA. Methods for evaluation of medication adherence and persistence using automated databases. Pharmacoepidemiol Drug Saf. 2006;15(8):565-574.

24. Ten Have TR, Localio AR. Empirical Bayes estimation of random effects parameters in mixed effects logistic regression models. Biometrics. 1999;55(4):1022-1029.

25. Kessler R, Partridge MR, Miravitlles M, et al. Symptom variability in patients with severe COPD: a pan-European cross-sectional study. Eur Respir J. 2011;37(2):264-272.

26. Sumner H, Woodcock A, Kolsum U, et al. Predictors of objective cough frequency in chronic obstructive pulmonary disease. Am J Respir Crit Care Med. 2013;187(9):943-949.

27. Fletcher C, Peto R. The natural history of chronic airflow obstruction. Br Med J. 1977;1(6077):1645-1648.

28. Agusti A, Calverley PM, Celli B, et al. Characterisation of COPD heterogeneity in the ECLIPSE cohort. Respir Res. 2010;11:122.

29. Barsky AJ, Peekna HM, Borus JF. Somatic symptom reporting in women and men. J Gen Intern Med. 2001;16(4):266-275.

30. Johnson KM, Bryan S, Ghanbarian S, Sin DD, Sadatsafavi M. Characterizing undiagnosed chronic obstructive pulmonary disease: a systematic review and meta-analysis. Respir Res. 2018;19(1):26. 


\section{Supplementary materials}

\begin{tabular}{|c|}
\hline $\begin{array}{l}\text { Cough } \\
\text { Since your last visit, do you usually cough when you don't have a cold? (Y/N) }\end{array}$ \\
\hline $\begin{array}{l}\text { Phlegm } \\
\text { Since your last visit, do you usually bring up phlegm from your chest, or do you usually have phlegm in your chest } \\
\text { that is difficult to bring up when you don't have a cold? }(\mathrm{Y} / \mathrm{N})\end{array}$ \\
\hline $\begin{array}{l}\text { Wheezing/whistling } \\
\text { Have you had wheezing or whistling in your chest at any time since your last visit? (Y/N) }\end{array}$ \\
\hline $\begin{array}{l}\text { Breathlessness } \\
\text { 1) Are you unable to walk due to a condition other than shortness of breath? (Y/N) }\end{array}$ \\
\hline $\begin{array}{l}\text { 2) Dyspnea (MRC dyspnea scale) } \\
\text { Level 1: Not troubled by breathlessness except with strenuous exercise. } \\
\text { Level 2: Troubled by shortness of breath when hurrying on level ground or walking up a slight hill. } \\
\text { Level 3: Walks slower than people of the same age on level ground because of breathlessness, or has to stop for breath } \\
\text { when walking at own pace on level ground. } \\
\text { Level 4: Stops for breath after walking about } 100 \text { yards }(90 \mathrm{~m}) \text { or after a few minutes on level ground. } \\
\text { Level 5: Too breathless to leave the house or breathless when dressing or undressing. }\end{array}$ \\
\hline
\end{tabular}

Figure SI Questionnaire used to measure symptoms at each study visit.

Abbreviation: MRC, Medical Research Council.

Table SI Characteristics of study participants in the subset of data that were used to assess dyspnea and any symptoms as 34 participants were unable to walk due to a condition other than shortness of breath

\begin{tabular}{|c|c|c|c|}
\hline & Visit I $(n=347)$ & Visit $2(n=24 I)$ & Visit $3(n=268)$ \\
\hline Age & $65.0(10.2)$ & $66.7(9.8)$ & $67.9(9.4)$ \\
\hline Male (vs female) & $54.5 \%$ & $54.4 \%$ & $54.1 \%$ \\
\hline BMI & $27.0(4.9)$ & $27.1(4.9)$ & $27.0(4.9)$ \\
\hline Caucasian (vs non-Caucasian) & $97.1 \%$ & $97.1 \%$ & $97.8 \%$ \\
\hline \multicolumn{4}{|l|}{ Comorbidities $^{\mathrm{a}}$} \\
\hline 0 comorbidities & $62.8 \%$ & $56.4 \%$ & $57.1 \%$ \\
\hline I comorbidity & $30.5 \%$ & $33.2 \%$ & $24.3 \%$ \\
\hline$\geq 2$ comorbidities & $6.6 \%$ & $10.4 \%$ & $18.7 \%$ \\
\hline Anxiety/depression (vs no) & $18.2 \%$ & $19.5 \%$ & $19.4 \%$ \\
\hline Smoking between visits (vs no) & $74.1 \%$ & $25.7 \%$ & $24.6 \%$ \\
\hline Lifetime pack-years smoked & $26.2(25.8)$ & $24.7(25.4)$ & $26.3(25.6)$ \\
\hline Any exacerbations ${ }^{\mathrm{b}}$ (vs no) & $7.5 \%$ & $10.0 \%$ & $14.6 \%$ \\
\hline $\mathrm{MPR}^{\mathrm{c}}$ & $76.9 \%(107.6)$ & $75.7 \%(111.9)$ & $76.5 \%(107.7)$ \\
\hline $\mathrm{FEV}_{1}(\mathrm{~L})$ & $2.2(0.8)$ & $2.1(0.8)$ & $2.1(0.7)$ \\
\hline$\%$ Predicted FEV, & $75.0(17.8)$ & $76.1(19.0)$ & $77.1(18.5)$ \\
\hline Diagnosed COPD (vs undiagnosed) & $23.3 \%$ & $27.0 \%$ & $32.5 \%$ \\
\hline Asthma (vs no) & $21.0 \%$ & $26.6 \%$ & $29.9 \%$ \\
\hline \multicolumn{4}{|l|}{ Symptoms (present vs absent) } \\
\hline Dyspnea & $53.3 \%$ & $46.5 \%$ & $44.8 \%$ \\
\hline Any symptoms & $83.9 \%$ & $77.2 \%$ & $74.6 \%$ \\
\hline
\end{tabular}

Notes: Means (SDs) are reported unless otherwise indicated. aParticipants reported whether they had ever been diagnosed with coronary artery disease, hypertension, diabetes, lung cancer, stroke, and tuberculosis at each study visit. ${ }^{b}$ COPD exacerbations of any severity (mild, moderate, severe) over the past 12 months. ${ }^{\mathrm{C}} 12$-month medication possession ratio for all respiratory-related medications.

Abbreviations: BMI, body mass index; MPR, medication possession ratio. 
International Journal of COPD

\section{Publish your work in this journal}

The International Journal of COPD is an international, peer-reviewed journal of therapeutics and pharmacology focusing on concise rapid reporting of clinical studies and reviews in COPD. Special focus is given to the pathophysiological processes underlying the disease, intervention programs, patient focused education, and self management protocols

\section{Dovepress}

This journal is indexed on PubMed Central, MedLine and CAS. The manuscript management system is completely online and includes a very quick and fair peer-review system, which is all easy to use. Visit $\mathrm{http}: / / \mathrm{www}$.dovepress.com/testimonials.php to read real quotes from published authors.

Submit your manuscript here: http://www.dovepress.com/international-journal-of-chronic-obstructive-pulmonary-disease-journal 